Revue européenne des migrations internationales

vol. $21-n^{\circ} 1 \mid 2005$

Femmes, genre, migration et mobilités

\title{
Articuler les rapports de sexe, de classe et interethniques
}

Quelques enseignements du débat nord-américain

\section{Christian Poiret}

\section{(2) OpenEdition}

\section{Journals}

Édition électronique

URL : https://journals.openedition.org/remi/2359

DOI : 10.4000/remi.2359

ISSN : $1777-5418$

\section{Éditeur}

Université de Poitiers

\section{Édition imprimée}

Date de publication : 29 mai 2005

Pagination : 195-226

ISBN : 2-911627-39-3

ISSN : 0765-0752

\section{Référence électronique}

Christian Poiret, «Articuler les rapports de sexe, de classe et interethniques », Revue européenne des migrations internationales [En ligne], vol. $21-\mathrm{n}^{\circ} 1$ | 2005, mis en ligne le 08 septembre 2008, consulté le 14 avril 2022. URL : http://journals.openedition.org/remi/2359; DOI : https://doi.org/10.4000/remi. 2359

Ce document a été généré automatiquement le 14 avril 2022

(c) Université de Poitiers 


\section{Articuler les rapports de sexe, de classe et interethniques}

Quelques enseignements du débat nord-américain

\section{Christian Poiret}

1 La création d'une autorité indépendante de lutte contre les discriminations annoncée par le Président de la république le 14 juillet 2003, a amorcé un débat public mené en particulier par les représentants des associations concernées. Pour le dire vite, l'enjeu de cette discussion, outre la question des moyens et des prérogatives d'une telle institution, concernait son champ d'intervention : pour des raisons d'efficacité, était-il souhaitable de constituer un organisme chargé de traiter l'ensemble des discriminations illégitimes telles qu'elles sont énumérées par les directives européennes relatives à l'égalité de traitement des personnes (à raison de la race, l'origine ethnique, le sexe, la religion, les convictions, le handicap, l'âge ou l'orientation sexuelle...) ou valait-il mieux se doter de plusieurs structures spécialisées. Ce débat a été tranché, au moins provisoirement, et cet article n'a pas pour objet de revenir sur ces questions tactiques. Il est plutôt l'occasion d'engager une discussion sur les difficultés théoriques que rencontrent ceux qui cherchent à articuler dans une même analyse, y compris et surtout lorsqu'elle est appliquée à des situations empiriques, plusieurs grands principes de stratification sociale, sans rester dans le cadre imposé par l'approche juridique qui donne un caractère d'actualité à cette thématique ${ }^{1}$. Plus précisément, nous nous intéresserons à la combinaison des rapports interethniques, des rapports de sexe et des rapports de classe, non parce qu'ils constitueraient les seules formes de catégorisation et de hiérarchisation sociales, mais parce que, au moins potentiellement, il n'est guère de domaine de la vie sociale qu'ils ne traversent ${ }^{2}$. De surcroît, à travers cette triade c'est bien à un cadre paradigmatique pouvant intégrer d'autres principes de classement et de hiérarchisation que nous souhaitons réfléchir.

Hormis à un niveau d'abstraction - et donc de généralité - assez élevé, cette question n'a guère été traitée de manière systématique en France. Les chercheurs qui se réfèrent à la sociologie des rapports interethniques - et nous nous incluons parmi eux affirment régulièrement que ceux-ci se combinent avec les autres rapports sociaux (de 
sexe, de classe, de générations) pour déterminer les hiérarchies sociales qui encadrent, habilitent et contraignent les acteurs sociaux. Ils rappellent rituellement que cette combinaison n'est pas une simple addition, mais force est de constater qu'ils peinent à en tirer toutes les conséquences et à rendre opératoire ce cadre théorique. Au mieux trouve-t-on, dans la production française, des travaux qui traitent conjointement de deux de ces dimensions et bien souvent de manière partielle et au prix d'une réduction des rapports interethniques au seul champ de la migration. Ainsi, tout un corpus, sociologique, ethnologique et historique, a-t-il été constitué depuis la fin des années 1950, concernant les rapports entre classe et ethnicité. Il est en grande partie composé d'ouvrages centrés sur la place de l'immigration dans la classe ouvrière et, dans la dernière période, de travaux relatifs au racisme dans le travail et l'emploi. Plus récemment - malgré quelques rares travaux pionniers à partir de la fin des années quatre-vingt - et en beaucoup moins grand nombre, des recherches ont abordé la relation entre genre et ethnicité, pour l'essentiel au travers de deux thématiques : celle des femmes en migration qui semble faire l'objet d'un fort développement ces derniers temps, et celle de la place dans l'espace public des descendantes d'immigré(e)s, mises à l'avant-scène par les violentes polémiques qui se sont développées autour de la question de la laïcité3.

3 Comparativement à la production scientifique française sur le sujet, la littérature nordaméricaine est foisonnante. Depuis une bonne vingtaine d'années, un riche débat académique et militant s'est développé, qui porte sur les aspects théoriques de l'articulation de la « race $^{4} »$, du genre et de la classe mais aussi sur ses implications politiques et juridiques, particulièrement dans le domaine de l'égalisation des chances et de la lutte contre les discriminations. Bien que les tenants et les aboutissants de cette vigoureuse discussion ne soient pas directement transposables d'une rive à l'autre de l'Atlantique, elle n'en est pas moins hautement instructive et ouvre des pistes de travail à ne pas négliger. Afin d'en tirer quelques leçons applicables au contexte français, nous allons tenter d'en donner un bref aperçu, forcément partiel et volontairement partial, en nous appuyant en particulier sur les travaux de féministes «noires " américaines qui ont mené ce débat de manière systématique et peuvent servir de point de référence pour tout un ensemble de travaux visant à articuler les dimensions de sexe (ou de genre), de classe et d'ethnicité (ou de «race») et portant sur d'autres groupes minoritaires (Asian-American, Latino-American...). Étant donné l'ampleur de la production sur le sujet, il ne s'agit évidemment pas d'un travail de recension systématique, mais plutôt d'une tentative d'introduction aux conditions d'émergence puis d'évolution de ce débat en Amérique du Nord. Le choix de cette délimitation géographique relève d'une commodité de présentation, afin de maintenir un minimum d'unité de contexte (scientifique, intellectuel, social, politique et juridique). Nous n'avons donc eu recours à des auteur(e)s européen(ne)s - en particulier britanniques, puisqu'eux aussi ont beaucoup écrit sur le sujet - que dans la mesure où ils intervenaient dans le débat américain.

\section{Tous les « noirs " ne sont pas des hommes, toutes les femmes ne sont pas «blanches " ${ }^{5}$}

Les principales initiatrices et animatrices de ce débat, et ce n'est pas un hasard, sont des femmes «noires", souvent issues de milieux populaires et ayant, pour certaines, 
bénéficié lors de leurs études supérieures, des dispositifs d'affirmative action mis en place à partir de la fin des années 1960, dans le prolongement du mouvement pour les droits civils.

La fin des années 1960 et les années 1970 ont été marquées par la contestation de la prétention à l'objectivité des approches académiques dominantes dans le champ des sciences humaines et sociales américaines, qualifiées de (néo)positivistes par les chercheuses féministes et par les chercheurs issus de minorités ethniques ou raciales(Hunter, 2002). Au-delà de la diversité de leurs références théoriques (marxisme, phénoménologie, post-structuralisme puis post-modernisme...) les unes et les autres s'accordaient à réfuter l'idée selon laquelle il y aurait une vérité objective qui pourrait être découverte en utilisant «la » méthode scientifique censée permettre au chercheur de se distancier de son objet de recherche en adoptant une posture axiologiquement neutre. En fait d'universalité, soulignaient-ils, les concepts, les cadres théoriques et catégories d'analyse correspondant aux canons académiques, sont issus pour l'essentiel de l'expérience du monde d'hommes, «blancs ", membres des " classes moyennes» et ils en ont, au moins pour partie, les limites. Une des bases épistémologiques des études féministes comme des études ethniques est donc que la connaissance est située [positional] et qu'elle reflète la perspective et les valeurs du producteur de connaissance ${ }^{6}$ [knower](Harding, 1991 ; Collins, 1990). En ce sens, la production savante dominante exprime la vision du monde du groupe dominant. C'est ce qu'illustre la formule enseignée à des générations d'écoliers selon laquelle "Christophe Colomb a découvert l'Amérique ». Ce caractère situé de la connaissance se retrouve aussi dans la manière dont les questions de recherche sont formulées. Il y a ainsi une différence entre se demander "pourquoi est-ce que les femmes gagnent moins» et "pourquoi est-ce que les hommes gagnent plus». Ces formulations alternatives identifient implicitement différentes sources du problème et elles vont donc inciter à réfléchir différemment (Rothenberg, 2002).

\section{La lutte pour la reconnaissance et la théorie de la connaissance située}

6 Il s'agissait donc de faire reconnaître l'existence des femmes et des membres des minorités ethniques ou raciales comme agents historiques dotés d'un point de vue particulier sur le social, enraciné dans une expérience spécifique de la vie quotidienne. Certaines féministes vont ainsi développer une théorie du "point de vue situé" (standpoint theory), une approche qui affirme que toute connaissance est située dans la position sociale et dans l'histoire de l'observateur comme dans celles de l'observé, et que cette situation doit être explicitée et faire l'objet d'une analyse réflexive (Smith, 1974, 1987, 1990; Hartsock, 1997). Parallèlement, un groupe de sociologues afroaméricains va proclamer la "mort de la sociologie blanche » dans un livre manifeste (Ladner, 1973) qui appelle à la fondation d'une sociologie « noire » basée sur des cadres d'analyse et des catégories issues de l'expérience vécue par les « Noirs ». Ces débats, loin d'être confinés au champ clos du monde universitaire, se développent aussi dans le cadre militant des mouvements féministes, communautaires et antiracistes. C'est également sur ces deux terrains interactifs qui se nourrissent l'un l'autre, que se développe, à partir des années soixante-dix, une critique de la critique avec l'émergence des Black Feminists ${ }^{7}$. Celles-ci reprochent aux féministes une vision globalisante des femmes conçue sur le modèle des «femmes blanches des classes 
moyennes » et aux spécialistes des relations raciales, militants ou universitaires, de considérer que tous les "Noirs", et par extension tous les membres des minorités ethniques ou raciales, sont des hommes. Dans les deux cas, disent-elles, ce sont les femmes « noires » et les spécificités de leur situation qui s'évaporent.

Les Black Feminists dénonçaient des tendances racistes au sein du mouvement des femmes états-unien remontant à ses origines, au XIXe siècle, lors de son développement dans le sillage de la lutte pour l'abolition de l'esclavage. Cette dénonciation était incarnée par la figure tutélaire de Sojourner Truth. Esclave affranchie, elle apostrophait ainsi les féministes «blanches" réunies lors d'une convention pour le droit des Femmes se tenant dans l'Ohio en 1851, en montrant les contradictions entre la définition de la féminité assignée aux femmes «blanches » nanties - et contestée par elles - et la réalité de la vie quotidienne des femmes afro-américaines :

«Les hommes disent que les femmes doivent être aidées lorsqu'elles portent des charges ou lorsqu'elles franchissent un obstacle et qu'elles doivent avoir la meilleure place partout. Personne ne m'a jamais aidé à porter des fardeaux ou à franchir une flaque de boue, ni ne m'a jamais donné la meilleure place. Et pourtant, ne suis-je pas une femme? (ain't a woman!) Regardez-moi. Regardez mes bras ! J'ai labouré et planté et cueilli, j'ai rentré des récoltes et aucun homme n'a pu me commander! Et pourtant, ne suis-je pas une femme? Je peux travailler autant qu'un homme et manger autant qu'un homme - quand j'en ai les moyens - et supporter le fouet autant qu'eux. Et pourtant, ne suis-je pas une femme? J'ai mis au monde treize enfants et j'ai vu la plupart d'entre eux réduits en esclavage et quand je hurlais ma plainte de mère, personne, hormis Jésus, ne m’a écoutée ! Et pourtant, ne suis-je pas une femme ${ }^{8}$ ?» (Loewenberg et Bogin, 1976, citées par Collins, 1986).

En déconstruisant la conception dominante de ce que devait être une femme au milieu du XIXe siècle, Sojourner Truth remettait en cause la vision patriarcale de la féminité et pointait les différences d'expériences et partant de revendications des femmes afroaméricaines, migrantes ou ouvrières.

De même, c'est dans le prolongement du mouvement des droits civiques et avec les mêmes ingrédients racisants que le mouvement féministe américain s'est renouvelé dans les années 1960. En février 1964, alors que, dans les ghettos, s'enchaînent émeutes et répression, le Congrès américain discute de l'adoption du Civil Right Act destiné à lutter contre les discriminations racistes et religieuses. Un parlementaire, démocrate conservateur $\mathrm{du}$ Sud, propose un amendement pour élargir ce texte aux discriminations à raison du sexe. Il s'agit au départ, d'une manœuvre prenant la forme d'un trait d'humour sexiste - accueilli avec hilarité par un Congrès composé quasiuniquement d'hommes - destinée à retarder l'adoption du texte et à en affaiblir la portée. Cet élargissement n'avait d'ailleurs été demandé ni par les (faibles) organisations féministes de l'époque, regroupées dans le National Women Party (NWP), ni par les rares femmes parlementaires. Cependant, dans le débat qui s'ensuivit, le NWP soutint cet amendement, relayé en cela par sa principale porte-parole au Congrès, Martha Griffiths. Celle-ci développa une argumentation soulignant l'analogie entre les situations minoritaires liées à la «race» et au sexe et affirmant que si les femmes «noires » étaient protégées par le Civil Right Act en tant que "noires", seules les femmes «blanches» se trouveraient dépourvues de protection face aux discriminations. De la sorte, Griffiths construisait une catégorie de "femme" constituée des seules femmes «blanches", par analogie avec la catégorie de "noirs» 
(hommes et femmes confondus), construction qu'elle exprimait clairement dans cette interpellation:

«Il me parait incroyable que des hommes blancs souhaitent placer la femme blanche dans une position aussi désavantageuse, bien que les hommes blancs l'aient déjà fait auparavant vos arrière grands-pères, prisonniers de leurs propres préjugés, ont permis à leurs ex-esclaves de voter, mais pas à leurs épouses blanches » (citée par Deitch, $1996:$ 300).

Comme le souligne Cynthia Deitch, ce type d'argument faisant appel au sentiment racial pour défendre les intérêts des femmes «blanches" était caractéristique du discours du National Women Party, des années 1940 jusqu'au milieu des années 1960, qui s'enracinait dans la rhétorique des organisations féministes du XIXe siècle. Cette utilisation de l'analogie entre la race et le genre, pour appuyer les revendications des femmes, en profitant de la dynamique du mouvement pour l'égalité raciale, amenait ces organisations, selon les circonstances, à exprimer d'un côté leur solidarité avec les femmes «noires » et, de l'autre, à faire appel au racisme "blanc » parfois accompagné d'antisémitisme et de xénophobie (Deitch, 1996 : 300). C'est pourtant en prenant appui sur cet amendement que la mobilisation des femmes s'est développée, permettant ainsi une "deuxième vague » du mouvement féministe américain, une renaissance sous la direction de "femmes blanches des classes moyennes", qui en ont d'ailleurs été les principales bénéficiaires dans un contexte de croissance économique. Et peut-être estce justement parce que ces femmes, en tant que membres de la classe et de la "race » dominantes, ne subissaient que la seule oppression sexiste, qu'elles disposaient des ressources nécessaires pour impulser ce mouvement.

À la fin des années soixante, l'élection de Richard Nixon confirme le déclin du mouvement des droits civils - annoncé dès 1965 par le fameux rapport Moynihan, conseiller du président démocrate Lyndon Johnson, qui désignait le «matriarcat pathologique » sévissant parmi les familles « noires » comme principale source de leurs maux - et amorce le dépérissement progressif des politiques d'affirmative action et de lutte contre la pauvreté qui n'a pas cessé depuis lors (Steinberg, 1997). C'est dans ce contexte que la domination "blanche " sur le mouvement des femmes est remise en question. Cela se manifeste à la fois d'un point de vue militant par la création d'organisations autonomes de femmes «noires » (et « chicanas ») et par une production théorique justifiant cette autonomie organisationnelle face aux tendances hégémoniques des «femmes blanches des classes moyennes » d'un côté et face aux discours machistes du mouvement nationaliste «noir » visant à renvoyer les femmes afro-américaines à la sphère domestique au nom d'une supposée tradition afrocentrique, de l'autre côté. C'est ainsi qu'en 1969, Frances M. Beal, dans un texte manifeste proposait le concept de "Double Jeopardy» (littéralement, il s'agit d'une double incrimination pour un même fait) pour désigner ce que signifiait être à la fois femme et "noire", concept qui a été étendu par la suite au fait d'être pauvre (Triple Jeopardy). En 1981, bell hooks', se référant à Sojourner Truth (Ain't a woman: Black Women and feminism), se livre à un travail de déconstruction de la catégorie "femme", pensée comme universelle, et de son homogénéité supposée qu'elle poursuit en 1984 dans son livre de référence Feminist Theory. From Margin to Center. Elle y polémique vigoureusement contre la tendance à l'appropriation du mouvement féministe par les femmes «blanches" des classes moyennes et supérieures, centrées sur leurs propres revendications et qui, malgré leurs proclamations formelles, ne prennent en compte ni le vécu des femmes noires ni celui des femmes pauvres. Globalement, elle insiste sur le 
lien entre la lutte contre le sexisme, le racisme et le classisme. hooks (1984: 4-5) conteste le discours féministe moderne qu'elle analyse comme fondé sur le fait que toutes les femmes sont opprimées et que des facteurs comme la classe, la race ou les préférences sexuelles ne créent pas une diversité d'expériences déterminant le poids $\mathrm{du}$ sexisme dans les vies individuelles des femmes. Selon elle, bien que beaucoup de femmes souffrent du sexisme, cela ne suffit par à forger une sororité, "un lien entre toutes les femmes ». Au contraire, les positions de classe et de race créent des différences de statut social, de style et de qualité de vie qui l'emportent sur l'expérience que les femmes partagent.

Plus encore, ces différences d'expérience engendrent des divergences de point de vue et d'objectifs pouvant aller jusqu'à l'antagonisme. Barbara Ellen Smith (1995) souligne ainsi que non seulement les femmes «blanches » bénéficient indirectement, par leurs liens familiaux, d'avantages liés à la dimension genrée et racisée de la domination de classe (le patronat et l'encadrement sont essentiellement composés d'hommes «blancs » qui sont aussi leurs maris, leurs pères...), mais que, de surcroit, en tant qu'employeuses de personnel domestique, elles se trouvent bien souvent en situation d'exploiter les femmes "noires » très nombreuses dans ce secteur d'activité. Elle en conclut ainsi que "c'est le paradoxe central du féminisme contemporain: ce n'est pas seulement que les identités de race, de classe, d'orientation sexuelle et autres facteurs génèrent des conflits parmi les femmes, mais que c'est le genre lui-même qui est source de différence » (Smith, 1995 : 685). C'est la manière même dont le genre (le sexe social) est construit, différentiellement selon la position de classe et de "race", qui se prolonge sous la forme d'un rapport d'exploitation entre employeuses et employées. De même, pour bell hooks :

«Les analyses féministes contemporaines de la famille impliquent souvent que le succès du mouvement féministe devrait conduire à l'abolition de la famille. Cette suggestion est très choquante pour beaucoup de femmes et particulièrement parmi les non-blanches. Tandis que les militantes blanches peuvent expérimenter la famille en premier lieu comme une institution oppressive (une structure sociale où elles font l'expérience de graves abus et de l'exploitation), beaucoup de femmes noires trouvent que la famille est la moins opprimante des institutions. En dépit du sexisme dans le contexte familial, elles peuvent y faire l'expérience de la dignité, de l'estime de soi et de l'humanisation qui ne sont pas expérimentées dans le monde extérieur où elles sont confrontées à toutes les formes d'oppression. [...] La dévalorisation de la vie de famille dans les discussions féministes, reflète souvent la nature de classe du mouvement. Les individus des classes privilégiées s'appuient sur de nombreuses institutions et structures sociales pour défendre leurs intérêts. Les bourgeoises peuvent répudier la famille sans croire que, ce faisant, elles vont perdre la possibilité de relations sociales, de sécurité et de protection » (1984 : 37).

Ce thème de la défense de la famille, comme structure de référence, revient souvent dans les ouvrages des "Black Feminists" mais on peut souligner qu'il n'est pas spécifique aux Afro-Américaines et qu'il se retrouve largement parmi les groupes privés d'accès au pouvoir d'État et aux instruments de la domination culturelle. Ici, il s'inscrit dans une tendance plus globale de ces auteures à réserver leurs critiques les plus sévères aux hommes «blancs » et aux femmes «blanches classes moyennes » et à ménager, relativement, les hommes «noirs », pour ne pas alimenter les représentations péjoratives de leur(s) communauté(s) ${ }^{10}$.

14 Pour hooks, du fait de la domination du féminisme par des femmes relativement privilégiées, la théorie féministe est incomplète, faute de prendre en compte la variété 
des expériences humaines. Afin de remédier à cette carence, les perspectives spécifiques des femmes minoritaires doivent sortir de leur marginalité et trouver place au cœur des recherches et du mouvement féministe [moving from Margin to Center]. D'un point de vue épistémologique et méthodologique, cette lutte pour la reconnaissance d'un point de vue spécifique des femmes minoritaires contre les biais « classe moyenne blanche" d'un côté et masculin de l'autre, va avoir (au moins) trois grandes conséquences : elle conduit à conférer une place centrale à l'expérience minoritaire ; elle implique une approche qui se veut anti-essentialiste et constructiviste des catégories de l'altérité ; elle met l'accent sur la nature combinée des différentes formes d'oppression ${ }^{11}$.

\section{Les conséquences théoriques et méthodologiques de la théorie de la connaissance située ${ }^{12}$}

La théorie du " point de vue » confère une place centrale à l'expérience qui se traduit par un avantage épistémique lié à la position minoritaire, conçu comme plus ou moins radical selon les auteur(e)s et leurs orientations théoriques. Celui-ci repose sur un double fondement lié, d'une part, à la position objective des dominées dans la structure sociale - une situation "d'outsider de l'intérieur ${ }^{13}$ " [Outsiders inside] - et, d'autre part, aux effets de type psychosociologiques qui en résultent et se manifestent sous la forme d'une "conscience dédoublée " [bifurcated Consciousness] issue de leur connaissance de la perspective des dominants et de celle des dominé(e)s sur le monde social.

Selon Patricia Hill Collins (1990), la concentration des femmes «noires» dans certains secteurs économiques et en particulier dans les emplois domestiques, leur a offert un point de vue sur les élites à partir de perspectives largement inaccessibles aux hommes «noirs » et aux intéressées elles-mêmes. Dans ce cadre, elles ont non seulement assumé des tâches domestiques mais aussi fréquemment tissé des liens forts avec les membres des familles dont elles s'occupaient. Il en a résulté un mélange de proximité - mettant à jour les contradictions du « pouvoir blanc » et contribuant ainsi à le démystifier - et de distance, dans la mesure où "ces femmes savaient qu'elles ne pourraient jamais appartenir à leurs "familles" blanches, qu'elles étaient des travailleuses économiquement exploitées et qu'elles resteraient donc des outsiders. Le résultat était une curieuse position d'outsider de l'intérieur, une marginalité bizarre qui a stimulé une perspective particulière », un point de vue unique des femmes «noires » sur elles-mêmes et sur la société. Certaines situations sociales sont donc plus appropriées que d'autres comme point de départ pour des projets de recherche qui tentent de comprendre les relations sociales de domination, d'autant que ces situations n'ont pas de réciproques - un Américain "blanc" peut n'avoir jamais eu directement affaire à un "Noir» au cours de son existence, alors que l'inverse est totalement impossible. Selon hooks, c'est parce qu'ils vivent à la fois à la marge et au centre de la société que les « Noirs » sont capables de voir les «Blancs» depuis une position avantageuse. Ils observent les «Blancs» sans être remarqués, ils les étudient avec un regard ethnographique [ethnographic gaze] (hooks, 1995). Cette observation n'est d'ailleurs pas gratuite puisqu'il en découle une connaissance qui peut être fort utile en cas d'hostilité ouverte, en rendant l'oppresseur plus prévisible et donc moins dangereux ${ }^{14}$.

Plus généralement, pour bell hooks (1984), les femmes noires, en tant que groupe, occupent une position sociale spécifique, non seulement parce qu'elles sont 
collectivement cantonnées dans les strates inférieures du marché du travail, mais aussi parce que leur statut social est plus bas que tous les autres et qu'elles subissent conjointement l'oppression raciste, sexiste et classiste. "Nous sommes le seul groupe qui n'a pas été socialisé pour assumer le rôle d'exploiteur/oppresseur en ce que nous n'avons pas "d'Autre" institutionnalisé à opprimer ou exploiter». Il y aurait donc là une source d'avantage épistémique qui conduit à mettre au centre de l'analyse sociale, les gens opprimés, ceux qui sont à la marge, afin de partir des points de vue susceptibles de fournir l'aperçu le plus critique sur ces relations d'oppression. De plus, ce type de socialisation des femmes afro-américaines fournirait un point de référence exceptionnel, quelque chose comme le zéro absolu de la domination, pour évaluer les inégalités. Alors que les femmes "blanches» aisées comme les hommes "noirs" auraient tendance à revendiquer les mêmes privilèges que les hommes «blancs », ce qui suppose de déplacer la ligne de clivage entre privilégiés et désavantagés, les femmes "noires" et pauvres seraient les seules à pouvoir porter de manière conséquente une perspective humaniste d'abolition de tous les rapports de domination et d'exploitation, constituant ainsi une sorte d'avant-garde de l'avant-garde des opprimés (hooks, 1981: 194). Pour déconstruire la catégorie "femme» supposée homogène, bell hooks tend ainsi à réifier les "femmes noires", considérées comme "pauvres» quasiment par essence. Ceci lui permet de faire de cette catégorie un puissant outil polémique qu'elle utilise pour ébranler les champs académiques et militants. La violence de ses interventions, probablement indispensable pour se faire entendre, a contribué à en faire une référence essentielle de ce débat.

Dans le prolongement de W.E.B DuBois (1969, [1908]) et de son concept de «double conscience ${ }^{15}$ ", certaines auteures (Smith, 1974, Collins, 1990) font usage de la notion de « conscience dédoublée " [bifurcated consciousness] pour qualifier la façon qu'ont les personnes marginalisées ou opprimées, d'expérimenter le monde social. Elles désignent sous ce terme la capacité à envisager les choses à la fois dans la perspective du dominant - qui est aussi la perspective dominante - et à la fois dans celle du dominé, ce qui ouvre la voie à une possible comparaison et à une déconstruction du contenu des catégories de l'altérité. Dans la mesure où les acteurs ont appris à voir le monde du point de vue des dominants, de ceux qui bénéficient de l'ordre social existant, cet ordre et ses hiérarchies leur semblent naturels. C'est dans un même mouvement que la perspective dominante construit les différences de "race", de genre, de classe comme des hiérarchies. Les membres d'une société anticipent certaines relations de domination et de subordination, ils s'attendent à voir certaines personnes en position de pouvoir et d'autres non. Dans le cours de la routine quotidienne, ceci va de soi, semble normal, naturel, intangible et universel. Aussi, les rapports de domination ne sont-ils pas spontanément perçus en tant que tels. Ils se présentent simplement comme la réalité de sorte que le racisme et le sexisme apparaissent comme invisibles ou comme inévitables. En fait, la catégorisation sociale est l'apanage du groupe dominant qui marque ceux qu'elle désigne $d u$ sceau d'une différence privative. Ce sont les minoritaires (les femmes, les « Noirs »...) qui sont vus comme « différents » tandis que les majoritaires (les hommes, les "Blancs»...) sont vus comme "normaux» (Rothenberg, 2002). Il s'agit donc, en partant du point de vue des opprimé(e)s, de contester toutes les conceptions anhistoriques et essentialistes des catégories de genre, de race, d'ethnicité... suivant ainsi l'exemple de Sojourner Truth.

19 Ce souci de déconstruire les catégories de genre a conduit certaines de ces auteures à considérer que le mouvement féministe «blanc», y compris dans ses tendances 
radicales et matérialistes, en utilisant le terme générique de «femmes » essentialisait cette catégorie. Ainsi Spelman (1988) considère que le genre ne peut pas être isolé des autres catégories d'identification que sont la "race» et la classe, ni le sexisme des autres rapports de domination, sauf à vouloir dissimuler la position sociale de celles qui se définissent comme des femmes avant tout. Dans cette perspective, les femmes afroaméricaines ne peuvent pas être seulement considérées comme des femmes «blanches" plus de la couleur ou encore des hommes «noirs » plus du genre. Pour comprendre leur condition sociale il faut donc analyser la combinaison inextricable de leur identité de "race", de classe et de genre. Aussi, dans l'espace ouvert par les vigoureuses interventions de bell hooks, vont se multiplier les travaux de femmes appartenant à des minorités raciales ou ethniques, centrés sur la nature combinée de l'oppression, ce que Patricia Hill-Collins (1990) appelle la « matrice de la domination».

Les prémices de ces approches sont que les axes d'oppression, comme le racisme et le sexisme, ne sont séparés ni conceptuellement ni en réalité, mais qu'au contraire, ils sont intrinsèquement interconnectés. De la sorte, toute identité raciale, par exemple, est expérimentée d'une manière genrée et, réciproquement, toute identité de genre est expérimentée d'une manière raciale particulière. Ainsi, Philomena Essed (1995 [1991]: 30-33) parle de "racisme genré » pour désigner l'oppression raciale des femmes "noires" en tant qu'elle est structurée par la construction raciste des rôles sexués issus du passé esclavagiste états-unien. L'utilisation sexuelle des esclaves "noires » combinée à leur exploitation, à la fois pour des travaux considérés comme spécifiquement féminins (domesticité) et pour les travaux manuels les plus durs (partagés par leurs homologues masculins), a eu un impact au-delà de l'abolition. Émancipées, les femmes "noires" ont continué à effectuer des tâches manuelles pénibles que ne faisaient pas les femmes «blanches». Il en a résulté la construction d'une féminité et d'une sexualité racisées spécifiques, à l'opposé de la féminité "blanche " des classes moyennes avec une femme "noire " pensée comme travaillant dur, forte, dominante et sexuellement dissolue. La restructuration du marché du travail dans les années 1950, liée en particulier à l'automatisation de l'industrie et au développement du secteur des services, gros consommateur de main-d'œuvre féminine, s'est traduite par une forte poussée du chômage parmi les hommes « noirs » peu ou pas qualifiés et par une croissance du nombre de mères célibataires, réactivant ainsi l'image de la matriarche noire. Ce stéréotype, qui combine des images racistes, sexistes et classistes, a renforcé la polarisation de genre dans la communauté noire : après avoir ignoré la nécessité pour les femmes «noires » de travailler hors de la maison, elles ont été accusées - dans le discours politique dominant comme dans le mouvement nationaliste noir - de prendre le travail des hommes « noirs » et de les aliéner en les privant de leur rôle de chefs de famille. Depuis cette époque, ces arguments ont été à la fois critiqués et régulièrement réactualisés pour blâmer les victimes et leur faire porter la responsabilité de leur situation infériorisée. Bien évidemment, le racisme genré ne concerne pas que les femmes. Aux stéréotypes féminins répondent ceux sur les hommes «noirs", vus comme des pères absents, des conjoints violents et exploiteurs et des violeurs potentiels.

21 Pour désigner, à un niveau macro-sociologique, le lien entre les différents systèmes d'oppression, Collins (1995: 492) utilise le terme d'oppressions entrecroisées [interlocking oppressions] c'est-à-dire " un modèle décrivant les structures sociales qui créent les positions sociales. [Alors que] la notion d'intersectionalité (intersectionality) décrit des processus microsociologiques, à savoir comment chaque individu et chaque groupe occupe une 
position sociale à l'intérieur des structures entrecroisées [...] qui modèlent conjointement l'oppression». Bien que le terme d'intersectionalité soit de loin le plus usité, d'autres universitaires ont proposé des concepts apparentés (co-synthèse, interconnectivité, multidimentionnalité, identité multiplicative...). Le postulat de départ de ces approches est que les différentes formes de domination ne sont pas séparées et additionnelles, mais qu'au contraire elles sont interactives dans leurs processus comme dans leurs effets. Ces formes de domination ne se limitent d'ailleurs pas au genre, à la classe et à la "race ». Collins ajoute la nationalité, l'âge, la religion et l'orientation sexuelle à son cadre théorique de la structure de la domination, ce qui permet de prendre en compte le fait que les mêmes personnes puissent simultanément être opprimées et oppresseurs, privilégiées et pénalisées. Dans cette optique, vivement discutée, aucune forme d'oppression n'est primaire bien que les individus et les groupes tendent souvent à définir l'une d'entre elles comme plus fondamentale que les autres.

\section{Les analyses de l'intersectionalité et les efforts d'application}

Si la nécessité de prendre en compte la diversité de position et d'expérience sociales des femmes n'a guère été contestée dans le champ des études féministes, certaines des analyses des Black Feminists (qui elles-mêmes ont des postulats théoriques divers) ont fait l'objet de critiques parmi lesquelles nous en retiendrons quelques-unes qui sont liées et nous semblent parmi les plus importantes pour notre propos.

Dans un article paru en 1991, Sherry Gorelyck, souligne les limites de l'empirisme féministe qui, en prenant comme point de départ le point de vue des femmes ordinaires et comme objet leur vie quotidienne pour la problématiser par une démarche inductive du type théorisation ancrée ${ }^{16}$, tend à confiner les enquêtes au monde de l'expérience. Poussée jusqu'à son terme, cette logique risquerait non seulement de morceler la recherche par enfermement dans chacun des milieux spécifiques étudiés, empêchant ainsi tout processus cumulatif, mais surtout elle nuirait à la compréhension du milieu étudié lui-même pour deux raisons : d'une part parce que «bien que l'oppression ne puisse être comprise qu'à partir du point de vue et de l'expérience de l'opprimé, l'organisation même du monde quotidien de l'oppression dans le capitalisme moderne occulte la structure de l'oppression »; et d'autre part, parce que cette structure de l'oppression n'est pas quelque chose de figé, mais qu'elle est faite de rapports sociaux incluant des contradictions sources de changement. Il en découle certaines conséquences: en premier lieu, les approches micro-sociologiques centrées sur le vécu quotidien doivent être replacées dans un cadre analytique plus large prenant en compte les grands rapports sociaux qui encadrent les interactions et leur donnent sens. Ensuite, parce que la vision de la classe, du groupe ethnique ou du genre dominant structure les relations matérielles, il est nécessaire de prendre également en compte le point de vue du groupe dominant, ne serait-ce que pour pouvoir le contester et lui opposer ceux des groupes dominés. Enfin, pour dépasser le morcellement de la connaissance, il s'agit de " découvrir non seulement les différentes expériences des différents groupes de femmes, mais aussi les processus qui créent ces différences [...] et comment ces processus - de racisme, d'impérialisme, de classe et d'oppression nationale, religieuse et sexuelle - sont connectés les uns aux autres et déterminent, selon des modèles très différents, la vie de chacun de nous ». 

[1994]) replace la question dans un cadre théorique plus large, en critiquant les courants postmodernes, illustrés par Spelman (1988), qui considèrent que l'affirmation selon laquelle les femmes constituent une catégorie sociale distincte serait une position idéaliste et essentialiste. Pourtant, souligne Juteau, le fait que les classes sociales soient divisées en fractions et traversées par les rapports de sexe et les relations interethniques, ne conduit pas à les considérer comme des constructions idéalistes ou essentialistes. Le problème vient de ce que, pour échapper au biologisme qui considère que les hommes et les femmes sont différents en nature, Spelman centre son analyse sur le genre et la construction culturelle des identités sexuelles. Or cette approche repose sur l'acceptation implicite de l'idée que les sociétés sont divisées naturellement en deux groupes sexuels (biologiques) sur lesquels se fonde le genre (le sexe social). Cette absence de remise en question du sexe - ainsi que de la "race» - esquive la question essentielle de la constitution des classes de sexe et des groupes raciaux. L'occultation de la construction sociale des catégories définies en termes biologiques de sexe (sexualisées) ou de " race » (racialisées) - conduit à leur naturalisation; elle les fait passer pour autonomes, masquant ainsi le fait qu'elles sont en fait interdépendantes (pas de «femmes» sans «hommes", pas de «Noirs» sans «Blancs »...) et construites dans des rapports sociaux de domination marqués par l'exploitation et l'appropriation d'humains. En comparant les différentes manières de théoriser l'articulation des rapports de sexe et d'ethnicité d'une part, de classe sociale et de rapports « raciaux » de l'autre, Juteau fait apparaitre des similitudes avec, dans les deux cas, des positions allant d'un certain réductionnisme, souvent d'inspiration marxiste (un des rapports sociaux, généralement de classe, détermine les autres qui sont alors généralement renvoyés à la sphère de l'idéologie) jusqu'à des modèles d'autonomie, plus ou moins relative, dans lesquels les différents rapports sociaux, bien qu'ils soient reliés les uns aux autres, sont analytiquement distincts.

position pour un modèle d'autonomie relative, Juteau, à l'instar de Stuart Hal (1986), met en garde contre deux types de réductionnisme dans l'analyse : horizontal et vertical. Le réductionnisme horizontal se focalise sur les catégories de "race", de genre ou de classe au détriment de l'ensemble des rapports sociaux qui les produisent, et il aboutit à une incapacité à conceptualiser ces différents types de rapports sociaux comme analytiquement distincts. Pour chacun de ces rapports, écrit-elle, il s'agit de comprendre dans quelles conditions un groupe accède à la domination sur un autre groupe et comment ce rapport se reproduit et se transforme dans le temps et dans l'espace. Le réductionnisme vertical ne prend pas en compte les niveaux économique, politique et idéologique et leurs médiations au sein de chacun de ces systèmes de domination. Il faut donc analyser l'instance matérielle de ces différents rapports sociaux, dans sa dimension économique mais aussi en tant que production des êtres humains, ainsi que ses dimensions politique, culturelle et idéologique, afin de comprendre comment se forment les collectivités et les identités ainsi que leur mobilisation dans les luttes des minoritaires. Au total, Juteau insiste sur le fait que les différences concrètes entre les femmes, les "Noirs ", les prolétaires... ne doivent pas masquer les rapports sociaux constitutifs de ces catégories dont le travail de dénaturalisation est loin d'être achevé.

Les tentatives de mises en œuvre de cette approche multidimensionnelle à partir de données empiriques, ont été finalement assez peu nombreuses, avec une «littérature sur 
le sujet qui reste surtout descriptive, et trop souvent descriptive de femmes d'une seule race et d'une seule classe " (Chafetz, 1997). Deux grandes pistes de travail ont cependant été explorées : l'une portant sur les effets inégalitaires de l'articulation des grands rapports de domination à une échelle macro-sociale, l'autre portant sur les mécanismes qui, dans les relations sociales, produisent ces effets à une échelle micro-sociale.

À une échelle macrosociologique, on peut noter différents travaux portant sur l'étude de la stratification sociale. Dans cette perspective, il s'agit non seulement de comparer la situation des différents groupes et sous-groupes en fonction d'indicateurs usuels comme le salaire moyen ${ }^{17}$, mais aussi de redéfinir les indicateurs de stratification de manière à prendre en compte les effets des différentes formes de hiérarchisation. Ferre et Hall (1996) proposent ainsi de repenser les modèles de stratification sociale en définissant celle-ci comme un processus régulant l'accès aux ressources, à l'autonomie - conçue comme liberté de mouvement et de choix de vie - et au pouvoir - comme participation aux processus décisionnels concernant le groupe social. Elles soulignent donc la nécessité d'inclure quatre dimensions supplémentaires à ces analyses : 1) celle de la violence et de la domination physiques qui limitent la marge d'autonomie, en matière d'accès à l'emploi ou de choix résidentiels par exemple, de ceux qui en sont victimes, qu'il s'agisse des violences sexistes ou de harcèlement raciste ; 2) celle de la relativité de la valeur de l'argent comme source et mesure du pouvoir économique puisque d'un côté, les salaires féminins considérés comme salaire d'appoint ne créent pas la même habilitation sociale, dans le cadre domestique comme dans celui des politiques sociales, tandis que de nombreuses recherches ont montré que les personnes ethnicisées ou racisées devaient payer plus cher que les majoritaires pour accéder à des services identiques (logement, assurances, prêts bancaires...); 3) celle de la stratification interne aux ménages qui fait que les hommes bénéficient d'avantages fournis par le mode de fonctionnement des familles «traditionnelles » qui favorisent notamment leur réussite professionnelle, alors que la situation économique des familles "non-blanches" ou des familles monoparentales est traitée comme si elle résultait de la déviance de ces types de ménage ; 4) celle du contrôle des hommes «blancs» sur le système politique qui joue un rôle important dans la définition des hiérarchies de genre et de « race ».

À un niveau microsociologique, on peut souligner l'importance de deux contributions, distinctes mais complémentaires, qui ont tenté de préciser des démarches d'enquête permettant d'appréhender l'articulation des différents modes d'infériorisation: celle de Philomena Essed et celle de West et Fernstermaker.

\section{L'expérience des dominé(e)s comme source d'information sur les processus structurants}

En comparant des femmes "noires », hautement qualifiées, aux États-Unis et aux PaysBas, Philomena Essed, bien qu'elle ne se positionne pas explicitement par rapport à la théorie de l'intersectionalité, ouvre des pistes de travail fort utiles pour concevoir comment le racisme, compris comme processus, se combine avec d'autres dynamiques de catégorisation par classe et par genre, pour structurer la société sur un mode conflictuel. Pour cela, elle présente un cadre d'enquête et d'analyse des données très précis et très opératoires. De plus, son choix d'une focalisation sur des femmes hautement qualifiées favorise son analyse de l'articulation avec les rapports de classe : 
en sortant du stéréotype de la femme noire et pauvre, elle montre comment ses interlocutrices sont renvoyées en permanence à une condition de classe inférieure à la leur et comment elles peuvent être concernées par les vexations et autres brimades infligées à leurs subordonné(e)s, racisé(e)s ou ethnicisé(e)s, dont elles sont régulièrement témoins.

Comme l'indique le titre de son ouvrage - Everyday Racism (1995 [1991]) - Essed s'intéresse au racisme quotidien, celui qui se manifeste banalement dans des pratiques familières, récurrentes, systématiques et qui exprime "l'entrelacement du racisme dans la trame du système social ». La notion de racisme quotidien permet de faire le lien entre les propriétés macrosociologiques du racisme (historiques, culturelles et structurelles) et les pratiques faites de micro-iniquités mais aussi de résistances qui contribuent à produire, à reproduire ou encore à transformer le système. Essed prend l'expérience subjective, telle qu'elle est rapportée par ses interlocutrices, comme point de départ pour développer son analyse théorique. Elle considère que ces récits du racisme banal ne sont pas de simples anecdotes mais qu'ils reflètent des "systèmes de connaissance " et des «systèmes de valeurs " qui composent un cadre de connaissance général du racisme, cadre mis en œuvre pour appréhender la réalité. En effet, sans cette connaissance générale, les événements rapportés ne pourraient pas être interprétés en termes de racisme. La notion d'expérience est donc centrale dans son travail, mais dans un sens large puisqu'elle comprend à la fois la connaissance, directe ou indirecte, d'événements spécifiques

- événements qui sont souvent tus ou présentés de manière avantageuse par les dominants - et la connaissance des phénomènes généraux du racisme qui donne sens à ces événements et fait le lien entre l'individuel et la structure sociale. Il s'agit donc de comparer les interprétations de la réalité par les différentes femmes interviewées, pour chercher des convergences, des régularités dans leur analyse de micro-événements afin d'en induire des processus structurants. Cette approche qui prend très au sérieux les récits des victimes du racisme suppose notamment la manifestation d'une capacité d'empathie de l'enquêteur. Or l'empathie suppose de pouvoir se mettre à la place de son interlocuteur ce qui, d'une part, ne va pas de soi et, d'autre part, va en l'encontre de toute une tradition sociologique très puissante dans laquelle le chercheur se situe en surplomb de son objet. C'est pourquoi Essed souligne le relatif avantage épistémique que constitue le fait d'appartenir aux mêmes groupes que les interviewé(e)s et se démarque du postulat de la nécessaire distanciation du chercheur d'avec son objet. À force de "faire avec» le racisme au quotidien, les minoritaires acquièrent une « expertise » qui leur permet de développer des attentes selon les circonstances. Pour cela, ils "activent des épisodes généralisés de ce type de situations ou 'scripts' [...] [qui] comprennent des connaissances socialement formées à propos des séquences d'événements, des règles et des conventions de comportement acceptables dans des situations spécifiques. Ces règles et conventions acceptables sont (sous) culturellement définies et, dans une large mesure, structurées par des conditions spécifiques de classe et de genre ". À partir de ces 'scripts', les minoritaires développent des scénarii du racisme qui comprennent des attentes à la fois quant à ce qui devrait se passer s'ils étaient traités "normalement» et quant aux risques de traitement racisé qu'ils sont susceptibles de subir. Ce sont bien ces scénarii qu'il convient d'identifier en tant que formes récurrentes d'actualisation du racisme.

Les récits du racisme quotidien vont donc présenter des structures communes: ils donnent le contexte d'un événement, les éléments de complication qu'il comporte, leur 
évaluation et leur interprétation comme étant, ou non, constitutifs d'incidents racistes à la lumière de la connaissance générale du racisme dont disposent les femmes interrogées. La comparaison entre les récits des afro-américaines aux États-Unis et ceux des migrantes surinamaises aux Pays-Bas, permet de montrer comment dans des contextes sociaux historiquement différenciés, le racisme s'actualise différemment et se combine de manière variable avec les rapports de genre et de classe. Ainsi, par exemple, la coïncidence entre rapports de classe et de "race » semble plus immédiate aux USA qu'aux Pays-Bas: alors que les femmes originaires du Surinam, refusent généralement de se situer en termes de classe, les Afro-Américaines insistent sur le fait que le racisme opère à travers les divisions de classe et que, globalement, être «Noir(e)» c'est être pauvre, et qu'être "Noir(e)» et pauvre revient à être traité différemment. De même, les formes genrées de racisme varient d'un pays à l'autre et leur signification également. Dans les deux cas, les femmes «noires » sont vues comme étant dotées d'une sexualité pathologique qui justifie leur harcèlement. Mais, aux USA, cette construction idéologique est plutôt associée à l'image de l'esclave ou de la servante «noire » qui peut être possédée par les «Blancs » et elle est analysée comme une fonction de contrôle des «Blancs » sur les « Noirs » à travers leurs femmes, tandis qu'aux Pays-Bas, elle aurait plutôt des connotations de sexualité "sauvage» et " exotique » pouvant être interprétée comme une spécification de la légitimation du contrôle généralisé masculin sur les femmes.

L'analyse développée par Philomena Essed reste cependant entravée par un cadre théorique qui l'amène à ne voir le racisme que comme un rapport qui met aux prises des « Noirs" et des "Blancs » : «le racisme est donc défini en termes de cognition, d'actions et de procédures qui contribuent au développement et à la perpétuation d'un système dans lequel les Blancs dominent les Noirs. Il faut rappeler que la domination raciste interagit avec les rapports de domination de genre et de classe ». Comme le souligne Hunter (2002), dans cette optique le racisme ne peut donc être exprimé qu'en termes de " Noirs » et de "Blancs » ou, à la rigueur, comme quelque chose de directement parallèle à cette expérience. Les limites de ce cadre paradigmatique qui ne permet pas de penser la construction des catégories de l'altérité elles-mêmes, apparaissent nettement lorsqu'il est utilisé pour comparer les migrantes du Surinam en Hollande et les descendantes d'esclaves étatsuniennes. La comparaison est justifiée par le fait que les unes et les autres sont des « noires », minoritaires dans des sociétés «blanches ». Pourtant, l'analyse des récits du racisme montre que les processus d'altérisation et d'infériorisation sont construits différemment de part et d'autre de l'Atlantique. En particulier, on peut voir que le rapport raciste états-unien, enraciné dans une longue histoire esclavagiste, tend à se focaliser sur des objectifs de ségrégation sociale et résidentielle qui visent à refuser le contact, sauf lorsque la hiérarchie « raciale » se superpose à la division hiérarchique du travail social, comme dans le cas de la domesticité; au Pays-Bas et dans le cas de migrantes, le rapport de domination est plus teinté d'ethnisme, avec de constantes injonctions à l'assimilation, tout en laissant une plus large place à la cohabitation sociale, y compris sous la forme d'unions «mixtes» puisque plus du tiers des Surinamaises interviewées ont un partenaire «blanc » et qu'elles ne s'en cachent pas.

\section{La réalisation situationnelle des catégories de l'altérité}

33 C'est à cette analyse de la production des catégories de l'altérité, manquante dans le travail d'Essed, que s'attachent Candace West et Sarah Fenstermaker dans leur article 
fameux "Doing difference» (1995a), en proposant une approche de type ethnomethodologique pour faire le lien entre les hiérarchisations sociales structurelles et les pratiques d'acteurs. Elles cherchent à comprendre comment les différents rapports de domination s'actualisent conjointement dans les interactions quotidiennes qui réalisent (rendent réelles) les catégories de genre, de « race » et de classe en fonction des contextes. Pour cela, elles proposent de concevoir les catégories de "race » et de classe, comme des processus, des "réalisations interactionnelles continues» [ongoing interactional accomplishments] sur le modèle de leur analyse du genre qui s'inscrit dans le prolongement des travaux de Garfinkel (1967) et de Goffman (1976) : dans les sociétés occidentales, nous considérons comme une évidence qu'il n'y a que deux sexes et nous agissons en conséquence dans le cours de nos interactions. Bien que le sens accordé aux catégories de sexe varie selon les circonstances, cette bipartition est expérimentée comme "naturelle», comme un donné objectif, indépendant des situations particulières dans laquelle elle est invoquée. Cette croyance est tellement partagée et institutionnalisée qu'à leur naissance, tous les êtres humains font l'objet d'une assignation sexuée qui va les amener à être classés comme mâles ou femelles, même lorsque les critères biologiques utilisés (et qui eux-mêmes varient historiquement et culturellement) sont contradictoires (hermaphrodisme, décalage entre les caractéristiques chromosomiques et la forme de l'appareil génital...). Le sexe est donc un construit social qui se poursuit continuellement dans la vie quotidienne, sous la forme d'un processus de catégorisation sexuée au cours duquel les apparences d'une personne sont interprétées comme si elles étaient indicatives d'une nature biologique supposée ${ }^{18}$. Sur la base de ce processus de catégorisation sexuée, le genre "est une réalisation située des membres sociaux, la gestion locale de conduites en relation avec les conceptions normatives des attitudes et des activités appropriées pour une catégorie sexuée particulière » (1995a : 21).

De la même manière, les catégories "raciales ", ethniques ou de classe peuvent être analysées comme des construits sociaux et en termes de processus. Les catégories « raciales » et ethniques changent et leurs significations, comme celles des catégories de genre, varient dans le temps et dans l'espace. "Ce qui est invariant, c'est la notion que les membres des différentes "races" ont des natures essentiellement différentes [et l'on pourrait ajouter, que les membres des différents groupes ethniques ont des cultures essentiellement différentes] qui expliquent leurs positions très inégales dans notre société" (1995a : 26). Les catégories de classe, quoiqu'étant moins tranchées et moins marquées du sceau biologique que celles de genre ou de "race », peuvent être analysées dans les mêmes termes. Bien que nos sociétés - et tout particulièrement la société américaine - s'affichent comme méritocratiques et ouvertes à toutes les mobilités sociales, on peut constater de puissants processus de reproduction sociale, même si celle-ci ne s'effectue pas terme à terme. Comme l'illustrent les discours sur la réussite ou l'échec scolaire, nous avons tendance à croire que la situation de classe d'une personne découle de ses qualités personnelles, de son ambition, de ses efforts, de ses mérites voire de ses dons ou de sa chance. Les hiérarchisations socio-économiques apparaissent ainsi normales, naturelles et quasiment immuables.

« Au total, la réalisation (accomplishment) [des catégories de l'altérité] consiste en la création de différences entre les membres des différentes [catégories] [...], différences qui sont ensuite utilisées pour maintenir les "distinctions essentielles" entre les identités catégorielles et les arrangements institutionnels qui les soutiennent » (1995a : 
25-26). Pour comprendre ce processus de réalisation des catégories de l'altérité, le grand apport de West et Fenstermaker est de mettre au centre de leur analyse le concept d'accountability formulé par les ethnométhodologues: d'une part, les membres d'une société doivent être capables de rendre compte de leurs comportements, ils doivent pouvoir se décrire eux-mêmes ainsi que leurs actions et les circonstances qui les environnent, "de manière sérieuse et conséquente ${ }^{19}$ "; d'autre part, les membres caractérisent routinièrement les activités auxquelles ils sont confrontés, en relation avec les conceptions normatives dominantes, et ils leur donnent une interprétation sociale en les situant dans le contexte d'autres activités, similaires ou différentes. Le mécanisme d'accountability est donc interactif: il permet aux membres d'orienter leurs activités, selon les circonstances, d'une manière compréhensible par les autres, de sorte qu'en retour, ceux-ci puissent reconnaître ces activités pour ce qu'elles sont en fonction du contexte. Cependant, ce processus qui permet de justifier et de comprendre une activité ne dépend pas seulement du libre choix des acteurs, soulignent-elles, il est à la fois interactif et institutionnel. Il s'inscrit dans des rapports sociaux et des cadres institutionnels qui l'environnent et qu'en retour, il contribue à reproduire. Le stock de connaissances communes aux membres le sens commun qui constitue un contexte de sens tacitement partagé - inclut des conceptions normatives dominantes relatives aux attitudes et aux activités appropriées selon les catégories ethniques ou raciales, conceptions qui reflètent la position structurelle des groupes catégorisés. Et, bien que l'accountability autorise les acteurs à adapter leur conduite aux circonstances, ils doivent tenir compte de ces normes, y compris pour les transgresser. C'est donc dans l'interaction avec les autres que les catégories de l'altérité (ethnique, de genre, de "race», de classe...) s'incarnent et se réalisent in situ, à travers ce double mouvement d'orientation et d'interprétation des conduites individuelles.

Quoique l'importance sociale, la saillance et les conséquences de chacun de ces types de catégorisation varient selon les situations, potentiellement, une personne altérisée peut être considérée comme comptable de n'importe laquelle de ses actions en tant que membre de chacune de ses catégories d'identification. De la sorte, son appartenance catégorielle peut être utilisée pour justifier ou discréditer cette action ou n'importe quelle autre. Puisque les acteurs savent qu'ils sont comptables de leurs actions, ils vont donc les concevoir de manière à ce qu'elles soient reconnaissables par les autres, en sachant que les différentes catégories dont ils relèvent constituent une ressource toujours potentiellement disponible pour leur interprétation. Cette disposition autorise à percevoir les attitudes et les activités des minoritaires comme découlant de leurs caractéristiques individuelles et à considérer leur position subordonnée dans l'ordre social comme étant normale, naturelle, cohérente avec leurs attributs catégoriels.

Cependant, parler d'actualisation des catégories de l'altérité dans l'interaction ne signifie pas forcément une correspondance des activités et attitudes des minoritaires avec les conceptions normatives dominantes de ce qu'elles devraient être. Cela indique seulement qu'elles peuvent toujours faire l'objet d'une évaluation en fonction de ces conceptions. De la non-coïncidence entre normes et pratiques, il peut résulter un certain désarroi des majoritaires et surtout des situations non-justifiables dans lesquelles les membres d'une catégorie particulière peuvent être vus et se ressentir comme n'étant pas à leur place. D'un autre côté, lorsque ces non-coïncidences se multiplient, elles peuvent être facteurs de changement et influer en retour sur le processus de catégorisation. West et Fenstermaker en concluent que «dans n'importe 
quelle situation donnée (du face à face au niveau "macro" des institutions), les réalisations simultanées de la classe, du genre et de la "race" vont différer dans leurs contenus et leurs effets. De situation en situation, la saillance des observations pertinentes pour la catégorisation (vêtements, couleur de peau...) peut sembler éclipser l'impact interactionnel de la réalisation simultanée des trois; néanmoins, [...] dans la mesure où le mécanisme de réalisation est partagé, leur réalisation simultanée est assurée " (1995a: 30). Et elles en tirent certaines implications pour la compréhension de l'articulation entre genre, classe et « race ». Tout d'abord, la pertinence de chacun de ces modes de classement pour l'action sociale dépend du contexte dans lequel ils sont accomplis et, en conséquence, ils peuvent être accentués ou atténués selon les cas. Ensuite, selon la manière dont la « race », le genre et la classe sont réalisés, une même activité peut avoir un sens très différent pour ceux qui y sont engagés comme l'illustrent les divergences d'appréciation sur la nature de la famille et de la fonction maternelle entre féministes «noires» et "blanches» (cf. supra). Enfin, les situations complexes dans lesquelles les relations de " race », de genre et de classe sont imbriquées, tendent à être étiquetées de manières variables et généralement restrictives (plutôt comme manifestation de racisme, de sexisme ou de domination de classe) selon les points de vue qui s'expriment.

\section{Les prolongements politiques et juridiques du débat}

Nous l'avons vu, dès son origine, le débat sur l'intersectionalité s'est développé conjointement dans les champs académique et militant. Dans ce dernier, les «Black Feminists » ont mené une critique systématique des stratégies féministes et antiracistes dominantes, incapables à leurs yeux, de prendre en compte l'expérience spécifique des "femmes de couleur». Cette démarche peut être illustrée par l'analyse que fait Kimberle Crenshaw (1994), de la place assignée aux femmes issues de minorités ethniques ou « raciales » dans les dispositifs de lutte contre les violences faites aux femmes et par les conséquences en termes de stratégies politiques qu'elle en tire.

\section{Une critique du féminisme et de l'antiracisme militants}

Pour Crenshaw, faute de capacité à penser l'intersection du sexisme et du racisme, les mouvements féministes et antiracistes tendent à se placer dans une situation de concurrence qui apparaît nettement lorsqu'il s'agit de lutter contre les violences faites aux femmes. Les antiracistes, soucieux de ne pas alimenter les nombreux stéréotypes raciaux sur le thème de la violence pathologique des "Noirs ", cherchent à éviter la question. Globalement, ils ont tendance à réduire les violences sexistes au sein des communautés ethniques ou "raciales " à une simple conséquence de la discrimination raciste contre les hommes, une manifestation de leur frustration face à leur privation de pouvoir dans les autres sphères de la société. Dans cette perspective, l'égalité entre hommes «noirs » et «blancs», suffirait à régler la question. De plus, les «femmes de couleur ", elles-mêmes, contribuent à minimiser le problème par leur réticence à signaler les violences à la police, par crainte d'introduire jusque dans l'espace domestique, les indignités subies par les minoritaires dans l'espace public. Les féministes apportent aussi leur contribution à l'occultation des violences faites aux " Noires ", fut-ce avec les meilleures intentions. Ainsi les campagnes de sensibilisation qui utilisent l'exemple de femmes «blanches classes moyennes» pour montrer que, 
contrairement à une opinion très répandue, cette question ne concerne pas que les familles pauvres et "de couleur ", arrivent simultanément à réifier et à effacer les femmes altérisées comme victimes. De même, certaines stratégies d'habilitation [empowerment] des femmes aboutissent à exclure une fraction de celles qui en ont le plus besoin. Crenshaw analyse le cas particulièrement choquant d'une migrante latinoaméricaine qui, confrontée à des violences conjugales, s'enfuit de chez elle avec son fils adolescent. N'étant pas admise dans les foyers gérés par des associations féministes, au motif qu'elle ne parle pas l'anglais et ne pourrait donc pas pleinement participer et bénéficier des échanges avec les autres femmes au sein de la communauté du foyer, elle se retrouve durablement à la rue.

Crenshaw en tire certaines conséquences en termes d'orientations stratégiques qui ne font sans doute pas l'unanimité, mais sont significatives des débats qui traversent les réseaux militants. Réfutant certaines interprétations post-modernistes, qu'elle taxe de " constructionisme vulgaire ", qui considèrent que "puisque toutes les catégories sociales sont construites, il n'y a pas de choses comme celles qu'on appelle 'Noirs' ou 'femmes', et qu'en conséquence cela a peu de sens de continuer à reproduire ces catégories en s'organisant autour d'elles", Crenshaw (1994) souligne au contraire l'importance de l'utilisation de ces catégories dans les rapports de pouvoir qui structurent la vie sociale. Certes, le processus de catégorisation est en soi un exercice de pouvoir dont l'initiative revient au groupe dominant. Mais ce processus n'est pas unilatéral et les personnes subordonnées peuvent y résister, notamment en subvertissant le sens des catégories par un retournement du stigmate. En ce sens, les revendications identitaires peuvent constituer des outils de résistance politique pour les membres de différents groupes subordonnés. La question se pose alors de savoir comment réintroduire la dimension multiple des identités, soulignée par les analyses intersectionnelles, dans les formes identitaires de mobilisation politiques. "Un début de réponse requiert que nous reconnaissions d'abord que les groupes organisés sur la base d'une identité dans lesquels nous nous trouvons nous-mêmes, sont en fait des coalitions, ou au moins des coalitions potentielles en attente d'être formées. [...] L'intersectionalité fournit une base pour re-conceptualiser la race comme une coalition entre des hommes et des femmes de couleur » (Crenshaw 1994). Il s'agit donc d'utiliser l'analyse intersectionnelle dans une perspective militante, pour appréhender les différenciations qui traversent les organisations «identitaires » et surtout pour négocier les formes d'expression de cette diversité au sein des coalitions politiques $^{20}$.

41 Cette perspective est cependant loin d'être simple à mettre en œuvre et elle continue à alimenter un débat nourri parmi les militants des organisations « identitaires ». Nancy Ehrenreich (2002) souligne ainsi quatre grands obstacles régulièrement rencontrés dans la réalisation de coalitions entre ces organisations : les conflits d'intérêts entre les différents groupes qui rendent difficile voire impossible leur défense simultanée; la tendance à la subdivision des différents groupes en sous-groupes autour d'une identité toujours plus restreinte, qui tend vers une individualisation des revendications; la difficulté, aggravée par cette fragmentation, de mesurer le degré d'oppression des uns et des autres de manière à déterminer des priorités en termes revendicatifs; et ce qu'elle appelle le problème du relativisme, c'est-à-dire le fait que si « chacun a à la fois le statut d'oppresseur et d'opprimé (selon les contextes), il devient impossible d'évaluer la légitimité des revendications d'oppression ou la valeur de propositions de réformes 
spécifiques $^{21} »($ Ehrenreich, 2002). Le débat se poursuit donc et certains universitaires militants tentent d'élaborer une théorie " post-intersectionnelle ».

\section{Intersectionalité et lutte contre les discriminations : l'approche juridique et la réification des catégories de l'altérité}

42 En raison de l'importance des dispositifs publics de lutte contre les discriminations en Amérique du Nord, les approches de type intersectionnel ont pris une importance particulière dans le domaine juridique. Elles ont notamment donné lieu, dans la deuxième moitié des années 1990, à l'émergence d'une école de pensée parmi les juristes universitaires états-uniens, le Critical Race Feminism, spécialisée dans l'étude des questions légales concernant les femmes, pauvres, membres de minorités ethniques ou « raciales» (Wing, 1997).

Cette approche s'inscrit dans la lignée des courants juridiques critiques [Critical Legal Studies] qui se sont développés à partir de la fin des années 1970 en opposition à la conception dominante de la loi comme étant neutre et objective et qui ont connu des débats similaires à ceux qui ont traversé les sciences sociales et les mouvements militants. Basées sur une démarche déconstructiviste visant à montrer comment la loi est utilisée pour perpétuer les hiérarchies de classe, de « race » et de genre, les Critical Legal Studies vont faire, elles aussi, l'objet d'une critique de la critique, qui met l'accent sur l'hégémonie des élites mâles et «blanches » dans l'orientation de ce courant. Il en résultera, à la fin des années 1980 , la création d'une nouvelle école, la Critical Race Theory, qui met au centre de son analyse les facteurs « raciaux» et vise à retourner l'outil législatif pour en faire un moyen de résistance et de libération et non plus d'oppression. Pour ses partisans, le racisme n'est pas une aberration mais une composante structurelle de la société américaine dont participe l'appareil juridique qui réifie et légitime les catégories «raciales» socialement construites. Dans cette perspective, les dispositifs de lutte contre les discriminations, du type Affirmative Action, n'ont pas pour objectif de remettre en cause cette structuration inégalitaire, mais de créer suffisamment d'exceptions aux privilèges «blancs» pour rendre plausible l'idéologie de l'égalité des chances. Il s'agit donc de refuser les prétentions au daltonisme volontaire [color blind] qui constituent la base du discours politique dominant depuis le début des années 1980, et de lui opposer une mobilisation politique et juridique centrée sur la « conscience de couleur » [colour consciousness] pour rectifier l'héritage des législations racistes.

En s'appuyant sur les analyses féministes de la jurisprudence, les fondatrices ${ }^{22} \mathrm{du}$ Critical Race Feminism vont se démarquer des théoriciens de la Critical Race Theory auxquels elles reprochent de présumer que l'expérience des femmes « de couleur » est semblable à celle des hommes "de couleur " mais aussi des juristes féministes auxquelles elles reprochent d'ignorer que la « suprématie blanche » est aussi le fait des femmes. Cette orientation va se traduire notamment par des travaux sur la jurisprudence en matière d'application des lois anti-discriminatoire, aux USA et au Canada, qui alimentent aujourd'hui la réflexion de certaines instances juridiques canadiennes.

À la fin des années 1980, Crenshaw (1989) analyse une série de jugements rendus par des cours états-uniennes qui refusent aux femmes «noires" le droit de se constituer comme plaignantes collectives dans des affaires de discrimination à l'emploi ${ }^{23}$. 
Pourtant, le titre VII du Civil Right Act, autorise un(e) plaignant(e) à mener une Class Action en matière de discrimination raciste ou sexiste, c'est-à-dire une procédure dans laquelle il ou elle représente l'ensemble des membres de sa catégorie. Certains jugements refusent ce droit aux femmes "noires» au motif que la loi prévoit des recours contre les discriminations racistes ou contre les discriminations sexistes, mais ne prévoit rien quand il s'agit des deux à la fois; d'autres jugements se fondent sur le fait que les femmes «noires » ne peuvent pas représenter la «classe » des femmes, ni celle des "Noirs", en raison des conflits d'intérêts entre les deux. Elles peuvent être représentées en tant que femmes ou en tant que « Noires » si une femme «blanche » ou un homme "noir » engage une Class Action, mais elles ne peuvent pas agir par ellesmêmes.

Prenant le travail de Crenshaw comme point de départ, Nitya Duclos (1993) analyse, au début des années 1990, l'ensemble des cas de discriminations à raison de la « race » ou du sexe portés devant les tribunaux canadiens sur la base de la loi sur les Droits de la Personne entrée en vigueur en 1985. Elle constate que l'occultation de l'existence des femmes appartenant à des minorités "raciales", en tant que groupe doté d'une expérience propre, est telle que, bien souvent, il est impossible de savoir si la plaignante appartient ou non à une minorité ou si un plaignant minoritaire est un homme ou une femme. Et, lorsqu'elles sont identifiables, rien n'indique qu'elles peuvent relever de cas différents des femmes «blanches » ou des hommes membres de groupes minoritaires; au contraire tout est fait pour que leur situation ressemble à celles des unes ou des autres. Pourtant l'analyse montre que, dans près d'un tiers des cas concernant clairement des femmes minoritaires, l'accusé a agi sur la base de stéréotypes mêlant la "race» et le sexe. Au total, comme l'avait déjà remarqué Crenshaw, les femmes racisées ou ethnicisées, qui devraient être au centre du dispositif de protection contre les discriminations, en disparaissent purement et simplement.

Les plaintes sont donc traitées comme des cas de discrimination soit à raison du sexe, soit à raison de la " race $^{24}$ ». Lorsque l'argument sexué est retenu, les juges font « comme si » les représentations stéréotypes sexuels ne variaient pas selon les groupes minoritaires et n'influaient pas sur les attitudes et les pratiques des harcèleurs ou des discriminateurs. Ils évitent ainsi de se poser la question de savoir si les variations de caractéristiques ethniques ou "raciales » des plaignant(e)s et des accusé(e)s ont une influence, aggravante ou atténuante, sur le préjudice subi par la victime. De même, lorsque c'est la dimension « raciale » qui est seule retenue, les magistrats font « comme si » le genre des protagonistes n'avait pas d'influence sur leur relation.

Duclos s'interroge quant aux causes de cette "disparition " des femmes membres de groupes minoritaires. Tout d'abord, elles «disparaissent» d'autant plus facilement qu'elles sont peu nombreuses à porter plainte et cela pour diverses raisons : ignorance de leurs droits; méfiance à l'égard du système judiciaire ; localisation professionnelle (par exemple, certaines provinces excluent les emplois domestiques du champ d'application de la loi) ; inadaptation des procédures, longues et incertaines, face à des discriminations dont l'effet est immédiat... Mais même lorsqu'elles portent plainte, leurs caractéristiques disparaissent des procédures et des jugements qui, selon Duclos, ne tiennent pas compte de la totalité de la personnalité de la plaignante. Tout est fait pour traiter les différents types de discriminations comme des phénomènes distincts : les discriminations à raison du sexe sont différentes de celle à raison de la "race » qui sont différentes de celle à raison de l'age... Ceci a pour conséquence de produire « des 
modèles essentialistes pour chaque type de discrimination qui excluent nécessairement certaines personnes" (Duclos, 1993: 40) et de ne pas prendre en compte les interactions complexes entre les différents facteurs qui constituent une part essentielle de la discrimination telle qu'elle est effectivement vécue. Ainsi, comment peut-on évaluer les différences de traitement sur la base de l'âge, sans prendre en compte les caractéristiques de genre et de « race » des protagonistes?

Le seul domaine où ces diverses caractéristiques sont prises en considération est celui de la détermination des compensations attribuées aux victimes, puisque les femmes des minorités « raciales » reçoivent plus que leurs homologues masculins (dans les cas de discrimination au logement en particulier) ou que les femmes du groupe majoritaire (dans les cas de harcèlement). Les juges ne sont donc pas totalement « aveugles ", mais leur manière de traiter les différentes caractéristiques des minoritaires comme étant indépendantes les unes des autres reflète en fait un point de vue particulier : celui du groupe dominant, qui se pense implicitement comme la référence par rapport à laquelle diffèrent les "Autres », celui du groupe privilégié qui ne fait pas l'expérience de la discrimination. "Les catégories sont séparées parce qu'elles représentent un ensemble de choix possibles divergents de la norme du groupe central. C'est lorsqu'on est au centre qu'on peut voir ces directions alternatives: par "race" (non-Blanc), ou par sexe (non-mâle), ou par religion (non-chrétien)... Ainsi conçues, les différentes formes de discriminations comprennent des présupposés implicites quant aux personnes qui peuvent les invoquer. La victime paradigmatique de discriminations racistes n'est pas un individu sans sexe, sans âge et dont la seule caractéristique est la "race", mais quelqu'un qui est conforme aux normes dominantes, et fait partie du "centre", sauf pour la "race" (Duclos, 1993 : 42) »; c'est donc, dans ce cas, un homme, chrétien, dans la force de l'âge et ne souffrant pas d'un handicap ${ }^{25}$. La victime type est quelqu'un qui ne diffère de la norme que par un aspect et c'est pour ce personnage que les lois anti-discriminations sont faites. Les plaignant(e)s qui s'en écartent par plus d'un aspect voient s'accroître le risque d'échouer dans leur procédure car les différences de traitements qu'elles subissent ne sont alors plus considérées comme de la discrimination mais comme une adaptation, une réaction à leurs (multiples) caractéristiques personnelles. De la sorte, la cause de la discrimination n'est plus située dans les relations sociales qui la produisent, mais dans les caractéristiques intrinsèques de l'individu. Ce type de traitement individualisant de la lutte contre les discriminations permet de négliger les causes structurelles et systémiques des processus discriminatoires. De plus, cette mise en parallèle de types de discrimination, étanches les uns par rapport aux autres, empêche aussi de penser «les situations dans lesquelles le plaignant et l'accusé appartiennent sous certains aspects au groupe dominant, mais pas sous d'autres et que c'est cette combinaison de pouvoir et d'oppressions relatifs qui oriente la discrimination " (Duclos, $1993:$ 47).

Sur la base de cette analyse critique, Nitya Duclos ouvre certaines pistes de travail pour repenser le dispositif de lutte contre les discriminations, en prenant en compte à la fois les caractéristiques des personnes concernées, plaignants et accusés (sexe, "race», âge...), leurs relations et les conduites qui en résultent, ainsi que le contexte social plus général au sein duquel elles se déroulent. Se référant aux travaux de Martha Minow (1990), elle plaide pour une approche en termes de relations sociales qui permet une utilisation dynamique, flexible et relationnelle des catégories de l'altérité en usage. Celles-ci ne devraient pas être de simples boîtes de classement, mais servir de point de départ pour tenter d'aboutir à "une compréhension aussi complète et complexe que possible de ce qui s'est passé, un compte-rendu qui reflète les différentes perspectives de ceux qui y sont 
impliqués, et pas seulement celle du groupe dominant» (Duclos, 1993: 50). Dans la confrontation à de multiples cas jurisprudentiels spécifiques, une telle démarche permettrait, selon Duclos, de reconceptualiser, de redéfinir et de raffiner les types de discriminations envisagés par la loi, ainsi que d'organiser méthodiquement le travail de comparaison qui se limite actuellement à l'exercice arbitraire d'estimation des dommages subis.

51 Ce type de réflexion juridique ne reste pas cantonné dans les cercles académiques ou militants et il nourrit aussi les analyses des instances judiciaires. Ainsi, la Commission Ontarienne des Droits de la Personne ${ }^{26}$ a-t-elle rendu public en 2001, un rapport sur l'usage de «l'approche intersectionnelle de la discrimination pour traiter les plaintes relatives aux droits de la personne fondées sur des motifs multiples ${ }^{27}$ ». On peut y lire que "l'approche intersectionnelle tient compte du contexte historique, social et politique, et reconnaît le caractère unique de l'expérience vécue en conséquence de l'intersection de tous les caractères pertinents. Elle permet de reconnaître l'expérience particulière de discrimination due à la confluence des motifs en cause et d'y remédier (p. 3) ». Il ne s'agit donc pas d'additionner des motifs de discrimination les uns aux autres pour en faire des circonstances aggravantes, mais de reconnaître que leur combinaison « produit quelque chose de différent (p. 7)».

Ce rapport reprend bon nombre des critiques formulées par Duclos (catégories de minorités " raciales» ou "visibles ») qui amalgament des individus et des groupes divers et incitent à considérer que seuls les membres du groupe dominant peuvent se comporter de façon discriminatoire; confusion entre "race», couleur, origine ethnique, lieu d'origine, ascendance... mélangés dans des catégories globalisantes) et il analyse les affaires dans lesquelles une approche intersectionnelle a été utilisée pour en tirer un constat de progrès par rapport aux modes de traitement antérieurs. Un des principaux enseignements de ce rapport est qu'il fait apparaître, à son insu, un risque de dérive dans l'utilisation de l'approche intersectionnelle. Placer au centre de la démarche juridique l'expérience vécue de la discrimination et son caractère unique peut s'avérer être une arme à double tranchant qui, à son tour, peut masquer les rapports sociaux qui produisent les processus discriminatoires. Lorsque la juge L'Heureux-Dubé, de la cour suprême du Canada argumente sur l'impact différentiel d'un acte discriminatoire selon la nature de la victime, elle fait appel à une analogie proprement réifiante : «Nul ne contesterait que deux projectiles identiques, lancées à la même vélocité, peuvent néanmoins laisser une marque différente selon le genre de surface atteinte. De la même manière, les groupes qui sont plus vulnérables sur le plan social, ressentiront les effets préjudiciables d'une distinction d'origine législative plus vivement que les groupes qui ne sont pas aussi vulnérables (p. 26)». Cette même tendance s'exprime crûment dans la conclusion du rapport: "Dans certaines affaires, des motifs comme le sexe, la race ou un handicap [...] peuvent se recouper et, ensemble, produire des effets uniques, créant des "minorités discrètes et insulaires" dont les caractéristiques constituent un handicap social ${ }^{28}$ (p. 32) ». En fait de démarche innovante, l'explication par le handicap social rappelle une vieille antienne, bien connue en sociologie de l'éducation par exemple, pour justifier la corrélation entre de piètres résultats scolaires et certaines caractéristiques socioculturelles des élèves et de leur famille. Cette notion idéologique permet de déplacer l'analyse des rapports sociaux et du mode de fonctionnement institutionnel qui produisent des situations d'échec fréquentes pour les dominés, vers les caractéristiques individuelles et collectives de ceux-ci, caractéristiques qui sont alors interprétées en termes de manques, d'absences, de carences. De même, poussée jusqu'à son terme le plus radical, l'approche juridique intersectionnelle risque d'aboutir à une 
individualisation complète des cas de discrimination qui les déconnectent, de fait, de toute logique sociale.

\section{Conclusion}

53 Au terme de cette présentation, que peut-on retenir de ces débats? Tout d'abord, et ce n'est pas secondaire, que la question de "l'intersectionalité » n'a pas été portée audevant de la scène par un mouvement du « haut » vers le «bas » - comme c'est le cas actuellement en France avec les initiatives institutionnelles liées au processus de construction européenne - mais par l'initiative des minoritaires, s'exprimant par ellesmêmes, mobilisées pour faire reconnaître leur existence en tant que groupe doté d'un point de vue spécifique. Comme l'écrivait Colette Guillaumin (1981) à propos des premiers écrits féministes, «ces textes minoritaires [...] sont à leur parution considérés à la fois comme légers et dangereux, comme plaisanterie de plus ou moins bon goût et menace. Mais, après, il n'est plus jamais question de poser les problèmes de la même façon qu'antérieurement ". L'expression de la colère des minoritaires remet en question l'évidence, elle oblige à reformuler les problèmes et à se doter de nouveaux outils théoriques. La théorie de la connaissance située, affirmant les spécificités de la vision du monde des femmes " noires ", incite à reconnaître le caractère partiel et partial de toutes les visions en sociologie (Juteau, 1981). Ceci ne signifie pas que le monde qui nous environne est un pur construit subjectif que chacun pourrait forger à sa guise et que, finalement, n'importe quel point de vue équivaudrait à n'importe quel autre. Tout au contraire, la théorie du point de vue devrait nous inciter à rechercher, derrière ces visions divergentes, les rapports sociaux qui les engendrent en produisant des groupes dont les intérêts sont (plus ou moins) contradictoires. À cet égard, les acquis théoriques des féministes matérialistes françaises - visiblement ignorés jusqu'à présent dans les débats des «Blacks Feminists »- devraient nous être fort utiles.

Pour ce qui concerne notre domaine de recherche, le racisme et l'ethnicité, contrairement à ce qui s'est fait en France jusqu'à présent, il convient donc de prendre au sérieux les témoignages des minoritaires comme source d'information privilégiée sur les processus de domination/subordination qui structurent l'ordre social. À travers ces récits, il est possible de comprendre comment les différents rapports de domination s'actualisent en se combinant. Encore faut-il éviter de se laisser enfermer dans une approche catégorielle qui postule l'existence de groupes identitaires au lieu d'en analyser les conditions d'émergence, de reproduction, de contestation et de transformation. Or l'impulsion du débat sur la question de "l'intersectionalité ", à l'occasion de la création d'un dispositif juridico-administratif est plutôt de mauvais augure à la lumière de la tendance récurrente (et peut-être inévitable) à la récupération des analyses en termes de rapports sociaux sous la forme d'analyses catégorielles, dès lors qu'elles sont saisies par le registre juridique et administratif du Droit et de l'État. Ce passage d'un cadre paradigmatique à un autre se traduit globalement par un mouvement de réification ou d'essentialisation des catégories de l'altérité qui tend, d'une part, à réduire les effets de la domination à la victimisation déniant ainsi aux minoritaires leur caractère d'agents historiques capables d'impulser un changement social - et, d'autre part, à imputer aux caractéristiques des dominés les inégalités qu'ils subissent. Face à cette dérive institutionnelle et bureaucratique, il n'est guère d'autre contrepoison qu'une re-sociologisation du débat et l'affirmation d'une 
approche relationnelle qui articule, de manière contextuelle et du macro au micro, les différents types de rapports sociaux.

\section{BIBLIOGRAPHIE}

ANDERSON Elizabeth (2003) Feminist Epistemology and Philosophy of Science, Standford Encyclopedia of Philosophy, http://plato.stanford.edu/entries/feminism-epistemology/

BEAL Frances M. (1969) Black Women's Manifesto ; Double Jeopardy: To Be Black and Female, consultable en ligne : http://www.hartford-hwp.com/archives/45a/196.html

CHAFETZ Janet S (1997) Feminist Theory and Sociology : Underutilized Contributions for Mainstream Theory, Annual Review of Sociology , Vol. 23, pp. 97-120.

CHANG Robert S, MACCRISTAL Culp Jerome(2002) After Intersectionality, UMKC Law Review, 71 (2), pp. 485-491.

COLLINS Patricia Hill (1986) Learning from the Outsider Within : The Sociological Significance of Black Feminist thought, Social Problems, 33 (6), pp. 14-32.

COLLINS Patricia Hill (1990) Black Feminist Thought: Knowledge, Consciousness, and the Politics of Empowerment, New York, Routledge, 335p.

COLLINS Patricia Hill (1995) Symposium on West and Fenstermarker's «Doing difference», Gender and Society, (4), pp. 491-494.

COMMISSION ONTARIENNE DES DROITS DE LA PERSONNE (2001) L'approche intersectionnelle de la discrimination pour traiter les plaintes relatives aux droits de la personne fondées sur des motifs multiples : http://www.ohrc.on.ca/french/ publications/ index.shtml

CRENSHAW Kimberlé W. (1989) Demarginalizing the intersection of race and sex ; a black feminist critique of discrimination doctrine, feminist theory and antiracist politics, University of Chicago Legal Forum, pp. 139-167.

CRENSHAW Kimberlé W (1994) Mapping the Margins: Intersectionality, Identity Politics, and Violence Against Women of Color, in Martha Albertson Fineman, Rixanne Mykitiuk, Éds. The Public Nature of Private Violence. New York, Routledge, pp. 93-118.

DEITCH Cynthia (1996) Gender, Race, and Class Politics and the Inclusion of Women in Title VII of the 1964 Civil Rights Act, in Esther Ngan-Ling Chow, Doris Wilkinson, Maxine Baca Zinn Éds., Race, Class \& Gender : Common Bonds, Different Voices, London, Sage Publications, pp. 288-307.

DUBOIS, William E. B (1969, [1908]) The Negro American Family, New York : Negro Universities Press.

DUCLOS Nitya (1993) Disappearing Women: racial minority women in human right cases, Revue Femmes et Droit, 6, pp. 25-31 Canada.

EHRENREICH Nancy (2002) Subordination and Symbiosis: mechanisms of mutual support between subordinating systems, UMKC Law Review, 71 (1). 
ESSED Philomena (1995 [1991]) Understanding Everyday Racism. An interdisciplinary Theory, London, Sage Publications, $321 \mathrm{p}$.

FERRE Myra Marx, HALL Elaine J (1996) Rethinking Stratification from a Feminist Perspective : Gender, Race and Class in Mainstream Textbooks, American Sociological Review, 61 (6), pp. 1-22.

GORELICK Sherry (1991) Contradictions of feminist methodologie, Gender and Society, 5 (4), pp. 459-477.

GARFINKEL Harold (1967) Studies in Ethnomethodology, Englewood Cliffs, NJ, Prentice-Hall.

GLAZER B, STRAUSS A. L (1967) The Discovery of the Grounded Theory. Strategies for Qualitative Research, New York, Aldine.

GOFFMAN Erving (1976) Gender Display, Studies in Anthropology of Visual Communication, 3, pp. 69-77.

GUILLAUMIN Colette (1977) Race et nature : Système des marques, idée de groupe naturel et rapport sociaux, Pluriel, 11, pp. 39-55.

GUILLAUMIN Colette (1978) Pratique du pouvoir et idée de Nature (1) : l'appropriation des femmes, Questions féministes, 2, pp. 5-30.

GUILLAUMIN Colette (1981) Femmes et théories de la société : remarques sur les effets théoriques de la colère des opprimées, Sociologie et société, 13 (2), pp. 19-31.

GUILLAUMIN Colette (2003 [1972]) L'idéologie raciste, Genèse et langage actuel, Paris Gallimard, coll Folio Essais.

HALL Stuart (1986) Gramsci's relevance for the study of race and ethnicity, Journal of communication inquiry, 10 (2), pp. 5-27.

HARDING Sandra (1991) Whose Science, Whose Knowledge? Thinking from Women's Lives, Ithaca, NY : Cornell University Press.

HARTSOCK Nancy C. M (1997) ‘Standpoint Theories for Next Century’ in S. J. Kenney and H. Kinsella Éds., Politics and Feminist Standpoint Theories, New York : Haworth Press.

HARTSOCK Nancy C. M (1998) The Feminist Standpoint Revisited and Other Essays, Colorado: Westview Press.

HERITAGE John (1984) Garfinkel and Ethnomethology, Cambridge, England : Polity.

HOOKS bell (1981) Ain't a woman: Black Women and feminism, Boston, South End Press.

HOOKS bell (1984) Feminist Theory: from margin to center, Boston, MA, South End Press.

HOOKS bell (1995) Killing rage: ending racism, 1st ed. New York : H. Holt and Co.

HULL Gloria, SCOTT Patricia B, SMITH Barbara [Éds] (1982) All the women are White, all the Blacks are men, but some of us are brave : Black Women's Studies, New York : Feminist Press at CUNY

HUNTER Margaret (2002) Rethinking epistemology, methodology, and racism : or, is White sociology really dead ?, Race and Society, 5 (2), pp. 119-138.

JUTEAU Danielle (1981) Visions partielles, visions partiales : visions des minoritaires en sociologie, Sociologie et société, 13 (2), pp. 33-47.

JUTEAU Danielle (1999) De la fragmentation à l'unité. Vers l'articulation des rapports sociaux, in L'ethnicité et ses frontières, Presses de l'Université de Montréal, pp. 103-129. 
LADNER, Joyce A. [Éd.] (1973) The Death of White Sociology, (collection of essays) New York : Random House.

LOEWENBERG Bert, BOGIN Ruth [Eds.] (1976) Black Women in Nineteenth-Century American Life. Their Words, Their Thoughts, Their Feelings, Pennsylvania State University Press.

MANN Susan A, KELLEY Lori R (1997) Standing at the crossroads of modernist thought : Collins, Smith, and the New Feminist Epistemologies, Gender \& Society, 11 (4), pp. 391-408.

MATHIEU Nicole-Claude (1991) Identité sexuelle/sexuée/de sexe ? in L'anatomie politique. Catégorisations et idéologies du sexe, Paris, Côté-Femmes, pp. 227-266.

MINOW Martha (1990) Making all the difference: inclusion, exclusion, and american law, Ithaca, Cornell University Press.

MYERS Kristen A, WILLIAMSON Passion (2001) Race talk: the perpetuation of racism through private discourse, Race \& Society, 4, pp. 3-26.

ROTHENBERG Paula S (2002) Learning to see the interrelatedness of race, class and gender discrimination and privilege: implications for policy and practice, communication consultable en ligne : http://www.e-quality.nl/assets/e-quality/publicaties/2003/lezingen.pdf

SMITH, Barbara E (1995) Crossing the great divides. Race, class and gender in southern women's organizing, 1979-1991, Gender \& Society, 9 (6), pp. 680-696.

SMITH Dorothy (1974) Women's perspective as a radical critique of sociology, Sociological Inquiry, 44, pp. 7-13.

SMITH Dorothy (1987) The everyday world as problematic. Boston, MA : Northeastern University Press.

SMITH Dorothy (1990) The Conceptual Practices of Power : A Feminist Sociology of Knowledge, Boston, Northeastern University Press.

SPELMAN Elizabeth V (1988) Inessential Woman: Problems of Exclusion in Feminist Thought, Boston, Beacon.

STEINBERG Stephen (1997) The liberal retreat from race since the Civil Rights Act, in M. Guibernau et J. Rex Éds., The Ethnicity Reader: Nationalism, Multiculturalism and Migration. Malden, MA: Polity Press, pp. 302-318.

WEST Candace, FENSTERMAKER Sarah (1995a) Doing Difference, Gender \& Society, 9, pp. 8-37.

WEST Candace, FENSTERMAKER Sarah (1995b) Symposium on West and Fenstermarker's « Doing difference ", Gender and Society, 9 (4), pp. 491-494.

WING Adrien Katherine [Éd] (1997) Critical Race Feminism : A Reader, New York University Press.

\section{NOTES}

1. Je tiens à remercier tout particulièrement François Vourc'h pour les pistes de travail, les orientations bibliographiques, les critiques et les remarques qu'il m'a généreusement dispensées et qui ont contribué à nourrir cet article. Merci également à ces lectrices exigeantes qu'ont été Véronique De Rudder, Danièle Juteau et Vijé Franchi.

2. Nous aurions pu y ajouter les classements par groupes d'âge et les rapports de générations qui présentent le même caractère d'ubiquité. Cependant ceux-ci offrent une caractéristique particulière : par définition, chaque individu passe par différentes classes d'age au cours de son 
existence, ce qui n'est pas forcément le cas des autres principes de classement et qui est même relativement rare dans le cas des catégories de « race » et de sexe.

3. Nous laissons volontairement de côté, pour le moment, la littérature sur la relation entre genre et classe qui a fait l'objet d'un débat spécifique au sein du mouvement des femmes en France.

4. Nous ne discuterons pas ici du paradigme des relations de " race ", nous contentant de constater qu'il constitue un cadre mental hégémonique aux USA. Nous utilisons les guillemets pour marquer le caractère de pure construction sociale de la notion de " race ", caractéristique qui ne diminue en rien son efficacité sociale.

5. Hull et alii, 1982.

6. Elles s'inscrivent ainsi dans une longue tradition sociologique et en particulier dans le prolongement des travaux de Marx et de Manheim auxquels les unes et les autres se réfèrent souvent en la circonstance.

7. Au nombre desquelles on peut citer Hazel Carby, Cathy Cohen, Patricia Hill Collins, Kimberlé Crenshaw, Angela Davis, Paula Giddings, bell hooks, Joy A. James, Nevill Irvin Painter, Barbara Smith, Michele Wallace, Patricia J. Williams.

8. L'ensemble des citations est librement traduit par l'auteur de l'article.

9. L'auteure a repris le nom d'une de ses aïeules, qui était esclave. Il s'écrit sans majuscule pour signifier le caractère approprié, réifié du statut d'esclave.

10. Cette configuration n'est pas sans ressemblance avec les prises de position des jeunes femmes qui revendiquent le port du foulard en France ou avec les débats qui ont entouré l'émergence du mouvement « Ni putes, ni soumises » dont l'action est prioritairement orientée vers des quartiers populaires au peuplement bien souvent ethnicisé ou racisé.

11. L'oppression étant définie par bell hooks comme une absence de choix pour ceux et celles qui en sont victimes.

12. Pour une discussion beaucoup plus approfondie de ces questions épistémologiques, cf. notamment, Anderson, 2003 ; Gorelick, 1996 ; Saltzman Chafetz, 1997 ; Mann \& Kelley, 1997 ; Harding, 1991.

13. Collins, 1986.

14. Pour une étude de cas originale explorant les possibilités de cet avantage épistémique, cf. Myers et Williamson, 2001.

15. La sensation «bizarre " d'être " à la fois un "Nègre" et un Américain ».

16. À propos de la théorisation ancrée, cf. Glazer et Strauss (1967).

17. Ainsi, selon Rothenberg (2003) au début des années 2000 aux USA, le salaire moyen des hommes noirs était inférieur de $25 \%$ à celui des hommes blancs, celui des femmes noires de $37 \%$, celui des hommes hispaniques de $38 \%$ et celui des femmes hispaniques de $48 \%$..

18. En cela, West et Fenstermaker rejoignent les analyses des féministes matérialistes françaises qui ont théorisé les rapports sociaux de sexe : Guillaumin (1977, 1978), Mathieu (1989).

19. Héritage John (1984) cité par West et Fenstermaker.

20. Pour une illustration appliquée de ce type de réflexion stratégique, confère par exemple, l'article de Barbara Ellen Smith (1995) qui décrit 20 années d'expérience de création et d'animation d'une association pluriethnique de lutte pour l'accès à l'emploi des femmes dans le Sud-Est des États-Unis.

21. Citée par Chang et MacCristal (2002: 486).

22. Parmi lesquelles on peut mentionner Kimberlé Crenshaw, professeur de droit à l'UCLA et à Columbia.

23. En particulier, De Graffenreid Vs General Motors, 1976.

24. En fait sous la catégorie de " race ", sont rangées des références aux caractéristiques phénotypiques, mais aussi au lieu de naissance, à la nationalité, à l'origine ethnique... parfois combinées entre elles de manière aléatoire. 
25. Duclos rejoint ainsi les analyses de Colette Guillaumin (2003 [1972]), qui défini « en creux » les caractéristiques du groupe dominant qui tend, généralement, à ne pas se définir et à se penser comme le référent universel par rapport auquel les « autres » diffèrent.

26. Organisme public indépendant du gouvernement, chargé de veiller à l'application du Code des droits de la Personne en Ontario, la Commission instruit les plaintes pour discrimination, effectue éventuellement une médiation entre les parties et met en œuvre des politiques de prévention.

27. Consultable en ligne sur le site de la Commission.

28. Souligné par nous.

\section{RÉSUMÉS}

La combinaison des rapports de sexe, de classe et interethniques dans la production des inégalités n'a été que très partiellement étudiée en France, et surtout de manière très générale et fort peu opératoire. En s'appuyant sur les travaux des Black Feminists, cet article propose une introduction au riche débat sur le thème de l'intersection de ces différents rapports sociaux qui s'est développé en Amérique du Nord, de manière conjointe dans les champs académiques et militants, depuis une trentaine d'années. Il aborde ses conditions d'émergence et de développement, sous l'impulsion d'Afro-américaines voulant faire reconnaître la spécificité de leur expérience du monde social ; les enjeux épistémologiques et méthodologiques ainsi que les efforts d'application empirique de ce cadre d'analyse; enfin ses prolongements juridiques et politiques, marqués par une tendance à la réification des catégories de l'altérité (la " race », le genre, la classe) au détriment des processus qui les produisent.

À l'heure où la France s'apprête à se doter d'une autorité indépendante de lutte contre les discriminations, cette vigoureuse discussion offre nombre d'enseignements tant pour leur analyse que pour leur traitement.

\section{Articulating Gender, Class and Interethnic Relations :The North American Debate}

The intersection of gender, class and interethnic relations in the production of inequalities has as yet only been partially examined in France, and, only in a very general and non-operant way. Drawing on the work of Black Feminists in the USA, this article proposes an introduction to the rich debate on the theme of the intersection of these different forms of social relations, as this developed over thirty years in North America, both in academic and militant spheres. The article addresses the conditions of emergence and of development of this intersectionality, under the impetus of Afro-Americans wanting to make known the specificity of their experience of the social world; the epistemological and methodological issues at stake as well as the efforts to empirically apply this analytic framework ; lastly, the legal and political implications, marked by the tendency to reify categories of otherness ("race", gender and class) to the detriment of the processes that generate them.

At a time when France is about to set up an independent authority to fight against discrimination, this vigorous discussion offers a number of interesting teachings, regarding both analysis and treatment of discriminations.

Articular las relaciones de sexo, de clase e interétnicas : varias enseñanzas del debate norteamericano 
El estudio de la combinación de las relaciones de sexo, de clase e interétnicas en la producción de desigualdades ha sido parcialmente abordado en Francia y de manera muy general y poco operacional. Apoyándose en los trabajos de las Black Feminists, este artículo propone una introducción al rico debate que desde hace treinta años, y de manera concomitante entre el mundo académico y militante, aborda el tema de la intersección entre las diferentes relaciones sociales que se ha desarrollado en América del Norte. Este debate analiza : las condiciones de su emergencia y desarrollo bajo la impulsión de Afro-Americanas deseosas del reconocimiento de la especifidad de su experiencia del mundo social ; las cuestiones epistemológicas y metodológicas así como los esfuerzos de aplicación empírica del marco de análisis; y, por último, las prolongaciones jurídicas y políticas marcadas por la tendencia a la reificación de las categorías de la alteridad (la « raza », el género, la clase) en detrimento de los procesos que las producen.En un momento en que Francia se dispone a dotarse de una autoridad independiente de lucha contra las discriminaciones, esta vigurosa discusión ofrece numerosas enseñanzas tanto por su análisis como por su tratamiento.

\section{INDEX}

Index géographique : Canada, États-Unis

Mots-clés : Américains, discrimination, femmes, rapports sociaux, relations entre les sexes, relations sociales

\section{AUTEUR}

\section{CHRISTIAN POIRET}

Sociologue, maître de conférences à l'Université de Haute-Bretagne, membre du CERTAC (Rennes 2) et de l'URMIS (CNRS-Paris 7) : poiret@paris7.jussieu.fr 